\title{
Changes in Soluble TWEAK Concentrations, but Not Those in Amyloid- $\beta$ (1-40), Are Associated with a Decrease in Carotid Intima-Media Thickness after Bariatric Surgery in Obese Women
}

\author{
Jesús M. Gómez-Martin ${ }^{a} \quad$ Enrique Aracil ${ }^{b} \quad$ María Insenser ${ }^{a}$ e \\ Gema de la Peñad, $f$ Miguel A. Lasunciónd, ${ }^{d}$ Julio Galindo ${ }^{c}$ \\ Héctor F. Escobar-Morreale ${ }^{a, e}$ José A. Balsa ${ }^{a, f}$ José I. Botella-Carretero ${ }^{a, f}$ \\ aDepartment of Endocrinology and Nutrition, Hospital Universitario Ramón y Cajal and \\ Universidad de Alcalá and Instituto Ramón y Cajal de Investigación Sanitaria (IRYCIS), \\ Madrid, Spain; ${ }^{b}$ Department of Vascular Surgery, Hospital Universitario Ramón y Cajal \\ and Universidad de Alcalá and Instituto Ramón y Cajal de Investigación Sanitaria (IRYCIS), \\ Madrid, Spain; ' Department of General and Gastrointestinal Surgery, Hospital Universitario \\ Ramón y Cajal and Universidad de Alcalá and Instituto Ramón y Cajal de Investigación \\ Sanitaria (IRYCIS), Madrid, Spain; ${ }^{\mathrm{d}}$ Department of Biochemistry Research, Hospital \\ Universitario Ramón y Cajal and Universidad de Alcalá and Instituto Ramón y Cajal de \\ Investigación Sanitaria (IRYCIS), Madrid, Spain; ${ }^{e}$ Centro de Investigación Biomédica en Red \\ Diabetes y Enfermedades Metabólicas Asociadas (CIBERDEM), Madrid, Spain; ${ }^{\dagger}$ Centro de \\ Investigación Biomédica en Red Fisiopatología de la Obesidad y Nutrición (CIBEROBN), \\ Madrid, Spain
}

\section{Keywords}

Soluble TWEAK · Amyloid- $\beta$ - Metabolic syndrome · Bariatric surgery · Carotid intima-media thickness

\section{Abstract}

Background/Aim: Soluble tumor necrosis factor-like weak inducer of apoptosis (STWEAK) and amyloid- $\beta(1-40)\left(A \beta_{40}\right)$ emerged as markers of cardiovascular risk because of their actions in the endothelium and their role in atherosclerotic progression. The aim of this study was to analyze the association of these two factors with the decrease in carotid intima-media thickness (cIMT) after bariatric surgery in obese women. Methods: We studied 60 severely obese women, of whom 20 were submitted to laparoscopic Roux-en-Y gastric bypass (RYGB), 20 to sleeve gastrectomy (SG), and 20 to lifestyle modification therapy. Circulating sTWEAK, A $\beta_{40}$, high-sensitivity $\mathrm{C}$-reactive protein, plasminogen activator inhibitor type 1 , insulin resistance 
(HOMA-IR), and CIMT were measured at baseline and after 1 year of follow-up. Results: sTWEAK increased similarly after both surgical procedures, whereas the increase observed after lifestyle intervention did not reach statistical significance. $A \beta_{40}$ showed no differences between groups of women, nor did it change during follow-up. The decrease in cIMT at 12 months correlated with the decrease in body mass index (BMI) $(r=0.45 ; p<0.001)$ and fasting insulin $(r=0.30 ; p=0.038)$, and also with the increase in sTWEAK ( $r=-0.43 ; p=0.002)$. Multivariate linear regression showed that only the changes in BMI $(\beta=0.389 ; p=0.005)$ and $\operatorname{sTWEAK}(\beta=-0.358 ; p=0.009)$ were associated with the decrease in CIMT $\left(R^{2}=0.313 ; F=\right.$ 9.348; $p<0.001$ ). Conclusions: One year after bariatric surgery, RYGB and SG induced a similar increase in circulating STWEAK that occurred in parallel to the decrease observed in CIMT.

(C) 2020 The Author(s)

Published by S. Karger AG, Basel

\section{Introduction}

Obesity is a major health issue with increasing worldwide prevalence [1,2] and is also a major risk factor for cardiovascular disease [3, 4]. Obesity surgery has decreased overall and cardiovascular mortality in long-term prospective studies [5-7]. In conceptual agreement, bariatric surgery also reduces the carotid intima-media thickness (cIMT) [8], a surrogate clinical endpoint widely used as a predictor of future stroke and myocardial infarction [9].

We have recently shown that both laparoscopic Roux-en-Y gastric bypass (RYGB) and sleeve gastrectomy (SG) decreased, to a similar extent, the cIMT in severely obese women presenting with a high cardiovascular risk [10]. Although weight loss was clearly associated with this beneficial effect, we failed to demonstrate any association with other well-known cardiovascular risk factors such as lipid profiles, oxidized lipoproteins, and blood pressure, or even with adiponectin, an adipokine linked to cardiovascular risk [10-12]. Nevertheless, a large body of evidence supports a key role of low-grade inflammation in the development of atherosclerosis and atherothrombosis through interactions with endothelium-dependent vasodilatation, platelet activation, atherosclerotic progression, and plaque rupture [13-15].

One of these inflammatory mediators is tumor necrosis factor-like weak inducer of apoptosis (TWEAK), which binds to its receptor, fibroblast growth factor inducible 14 (Fn14) [16]. Both TWEAK and Fn14 are expressed in the arterial wall [17], participating in different stages of atherosclerotic progression and subsequent plaque rupture [18]. Soluble TWEAK (sTWEAK), a product derived from the full-length membrane-bound TWEAK molecule, also binds to arterial Fn14 [19]. Under pathological arterial conditions such as atherosclerosis, Fn14 expression is upregulated [17], favoring sTWEAK binding and, consequently, decreasing its circulating levels [18]. Therefore, low levels of sTWEAK are found in different atherosclerotic states such as stable coronary artery disease or peripheral artery disease [20,21].

Amyloid- $\beta(1-40)\left(A \beta_{40}\right)$, a proteolytic fragment of the amyloid precursor protein, also plays a role in cardiovascular disease through its proinflammatory and prothrombotic properties [22, 23]. High levels of circulating $A \beta_{40}$ may impair endothelium-dependent vasodilatation [24] and stimulate platelet activation [25], facilitating plaque rupture. In fact, increased circulating levels of $A \beta_{40}$ are associated with an increased risk for cardiovascular mortality in patients with coronary artery disease [26,27].

The aim of the present study was to investigate the possible associations of a decrease in cIMT in obese women after obesity surgery with circulating sTWEAK and $A \beta_{40}$ concentrations, as well as with other well-known surrogate markers of systemic inflammation and fibrinolysis such as C-reactive protein (CRP) and plasminogen activator inhibitor type 1 (PAI-1).

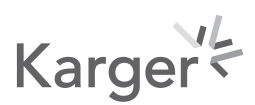




\section{Subjects and Methods}

\section{Patients and Study Design}

Sixty female candidates for obesity surgery presenting with a high cardiovascular risk, as defined by the presence of the metabolic syndrome, were included in the study. Of them, 20 were submitted to laparoscopic RYGB and 20 to SG. Another 20 women, who declined surgery and were submitted to diet and lifestyle modification, composed the control group. The groups were matched for age and cardiovascular risk.

For each individual patient, the choice of the surgical technique followed international guidelines for obesity surgery and our hospital's protocol, precluding randomization. This protocol allocates patients with a body mass index (BMI) above 45 preferentially to RYGB. The main characteristics of the RYGB procedure included a 20 - to $40-\mathrm{cm}^{3}$ gastric pouch, a biliopancreatic limb measuring 80-100 cm from Treitz' ligament, and a 120- to $200-\mathrm{cm}$-long alimentary limb. SG was performed with a laparoscopic linear stapler calibrated with a 32-Fr orogastric tube.

The diagnosis of the metabolic syndrome followed the criteria of the American Heart Association and National Heart, Lung, and Blood Institute [28], and required the presence of three or more of the following criteria: central obesity with a waist circumference $\geq 88 \mathrm{~cm}$, triglycerides $\geq 150 \mathrm{mg} / \mathrm{dL}$, blood pressure $\geq 130 / 85 \mathrm{~mm} \mathrm{Hg}$, fasting glucose $\geq 100 \mathrm{mg} / \mathrm{dL}$, high density lipoprotein cholesterol $\leq 50 \mathrm{mg} / \mathrm{dL}$, a previous diagnosis of type 2 diabetes mellitus, or treatment for hypertension or lipid disorders.

Exclusion criteria included mental impairment, an uncontrolled psychiatric condition or active substance abuse, active neoplastic disease, pregnancy, unstable or incurable serious preexisting comorbidities, and active treatment with thiazolidinediones.

Both the patients and the controls were evaluated at baseline and 1 year after surgery or after conventional treatment with diet and lifestyle modification, respectively. The participants were also reevaluated 6 months after surgery for anthropometric and analytical assessment (but not for cIMT measurements). From 2 controls and 3 patients submitted to SG, serum samples at 1 year of follow-up were not available for sTWEAK and $A \beta_{40}$ measurements; these missing data were handled by carrying forward the latest observation in an intention-to-treat fashion. Data on change in cIMT have been reported elsewhere [10].

\section{Assays}

Serum STWEAK was measured in duplicate by ELISA using a commercial kit (Human TWEAK Instant ELISA; Bender MedSystems, Vienna, Austria) with a sensitivity of $9.7 \mathrm{pg} / \mathrm{mL}$ and intra- and interassay coefficients of variation (CVs) of 7.9 and $9.2 \%$, respectively. Serum $\mathrm{A} \beta_{40}$ was measured with a commercial ELISA kit (Thermo Fisher Scientific, Waltham, MA, USA) with a sensitivity of $6 \mathrm{pg} / \mathrm{mL}$ and intra- and interassay CVs of 2.5 and $3.8 \%$, respectively. High-sensitivity CRP (hsCRP) was measured with a high-sensitivity colorimetric latex immunoassay (MULTIGENT CRP Vario Assay; Sentinel CH, Milan, Italy) using the ARCHITECT ci8200 analyzer (Abbot Diagnostics, Maidenhead, UK). PAI-1 was measured with an ELISA kit (R\&D Systems, Abingdon, UK) with a sensitivity of $0.06 \mathrm{ng} / \mathrm{mL}$ and intra- and interassay CVs of 8.0 and $6.3 \%$, respectively. Serum glucose levels were measured with standard colorimetric methods, using the ARCHITECT ci8200 analyzer. Fasting insulin was measured by immunochemiluminescence (Immulite2000; Siemens Healthcare Diagnostics, Caernarfon, $\mathrm{UK}$ ), with a $\mathrm{CV}<10 \%$. Insulin resistance in the fasting state was estimated by homeostasis model assessment (HOMA-IR), as previously reported [10].

\section{Statistics}

All variables are expressed as mean \pm SD unless otherwise indicated. The KolmogorovSmirnov statistic was applied to continuous variables, and logarithmic transformations were

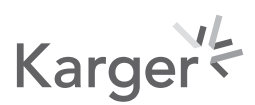


Gómez-Martin et al.: sTWEAK and cIMT after Bariatric Surgery

Table 1. Baseline characteristics of the included women and changes after follow-up

\begin{tabular}{|c|c|c|c|c|c|c|}
\hline & \multicolumn{2}{|c|}{ Controls $(n=18)$} & \multicolumn{2}{|l|}{$\mathrm{SG}(n=20)$} & \multicolumn{2}{|l|}{ RYGB $(n=20)$} \\
\hline & baseline & after 1 year & baseline & after 1 year & baseline & after 1 year \\
\hline Weight, kg & $105.9 \pm 15.9$ & $102.7 \pm 17.0$ & $109.9 \pm 13.4$ & $78.0 \pm 10.7^{*, \dagger}$ & $122.1 \pm 18.7^{\dagger, \ddagger}$ & $81.0 \pm 10.5^{*, \dagger}$ \\
\hline BMI, $\mathrm{kg} / \mathrm{m}^{2}$ & $41.8 \pm 6.0$ & $40.5 \pm 6.4$ & $43.0 \pm 4.0$ & $30.7 \pm 4.4^{*, \dagger}$ & $47.4 \pm 6.4^{\dagger, \ddagger}$ & $31.5 \pm 3.5^{*, \dagger}$ \\
\hline Fasting glucose, mg/dL & $108.4 \pm 16.4$ & $107.0 \pm 25.7$ & $114.3 \pm 33.8$ & $91.6 \pm 16.7^{*, \dagger}$ & $111.3 \pm 24.3$ & $84.4 \pm 6.7^{*, \dagger}$ \\
\hline Fasting insulin, $\mu \mathrm{IU} / \mathrm{mL}$ & $14.8 \pm 10.0$ & $15.4 \pm 0.5$ & $15.4 \pm 10.4$ & $7.0 \pm 3.6^{*, \dagger}$ & $25.0 \pm 13.9^{\dagger, \ddagger}$ & $4.2 \pm 2.4^{* \dagger, \ddagger}$ \\
\hline HOMA-IR & $3.9 \pm 2.6$ & $3.9 \pm 2.8$ & $4.3 \pm 4.5$ & $1.5 \pm 1.2^{*}$ & $6.8 \pm 3.9$ & $0.7 \pm 0.5^{*, \dagger, \ddagger}$ \\
\hline cIMT, mm & $0.74 \pm 0.14$ & $0.74 \pm 0.14$ & $0.73 \pm 0.13$ & $0.62 \pm 0.12^{*, \dagger}$ & $0.76 \pm 0.15$ & $0.68 \pm 0.12^{*, \dagger}$ \\
\hline
\end{tabular}

Data are mean differences \pm SD. SG, sleeve gastrectomy; RYGB, Roux-en-Y gastric bypass; BMI, body mass index; HOMA-IR, homeostasis model assessment of insulin resistance; cIMT, carotid intima-media thickness. ${ }^{*} p<0.05$ for the difference from baseline to the end of study; ${ }^{\dagger} p<0.05$ for the difference vs. controls; ${ }^{\ddagger} p<0.05$ for the difference vs. SG.

applied as needed to ensure normal distribution of the variables. Comparisons of continuous variables before and after bariatric surgery used repeated-measures general linear models, in which subject group (controls, RYGB, or SG) was introduced as a between-subjects effect. Differences between more than two groups were analyzed by one-way ANOVA followed by the post hoc Tukey test for multiple comparisons in case the ANOVA indicated a statistically significant difference. For nonparametric variables, the Kruskal-Wallis test followed by MannWhitney U tests were employed. Pearson's correlation method was performed to explore possible associations between the cIMT decrement and the absolute changes in other quantitative variables at the end of follow-up. Backward multivariate regression analysis was used to assess the predictors of change in cIMT; both independent and dependent variables were introduced into the model as the increment at the end of follow-up from baseline. All of the statistical analyses were performed using the SPSS 18.0 (SPSS, Chicago, IL, USA) statistical package.

\section{Results}

All women submitted to bariatric surgery were available for the analysis after 1 year, but 2 women in the control group were lost to follow-up. The women submitted to RYGB were 48 \pm 9 years of age, those submitted to SG $46 \pm 9$ years, and the controls $52 \pm 7$ years. As expected from our surgical protocol, the women submitted to RYGB had a higher BMI, fasting insulin, and HOMA-IR than those submitted to SG and the controls (Table 1). The baseline sTWEAK concentrations were significantly lower in the RYGB group than in the SG and control groups. On the other hand, there were no differences in baseline $\mathrm{A} \beta_{40}$, PAI-1, hsCRP, and cIMT (Table 1; Fig. 1).

When the patients were reevaluated after 1 year of follow-up, both SG and RYGB had induced higher decreases in BMI compared with lifestyle intervention, and also higher reductions in fasting insulin and HOMA-IR (Table 1). Circulating STWEAK concentrations had increased after bariatric surgery, regardless of the surgical technique applied, whereas no significant changes were observed in the controls submitted to conventional treatment (Fig. 1). PAI-1 and hsCRP concentrations had decreased after bariatric surgery, and both surgical techniques showed differences from the controls (Fig. 1). No changes were observed for $\mathrm{A} \beta_{40}$ concentrations (Fig. 1).

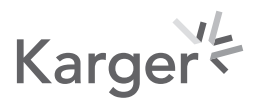




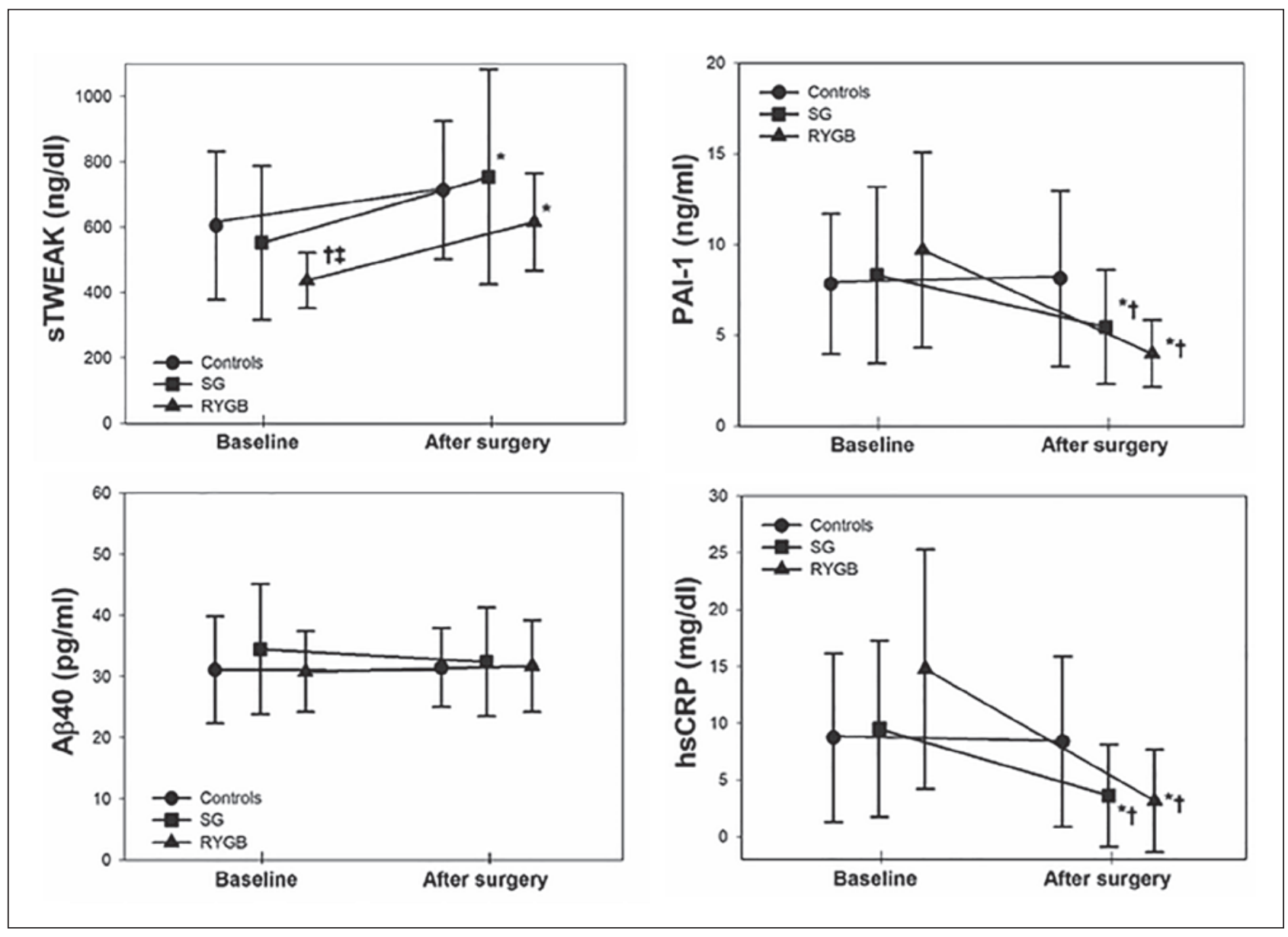

Fig. 1. Changes in STWEAK (soluble tumor necrosis factor-like weak inducer of apoptosis), amyloid- $\beta(1-40)$ $\left(\mathrm{A} \beta_{40}\right)$, plasminogen activator inhibitor type 1 (PAI-1), and high-sensitivity C-reactive protein (hsCRP). ${ }^{*} p<$ 0.05 for the difference from baseline to the end of study; ${ }^{\dagger} p<0.05$ for the difference vs. controls; ${ }^{\ddagger} p<0.05$ for the difference vs. SG. SG, sleeve gastrectomy; RYGB, Roux-en-Y gastric bypass.

When considering the results in all patients and controls together, we found a positive correlation between the decrease in cIMT and the decrease in BMI $(r=0.45 ; p<0.001)$ and in fasting insulin $(r=0.30 ; p=0.038)$. Besides, cIMT was inversely correlated with the increase in STWEAK $(r=-0.43 ; p=0.002)$, but showed no statistically significant correlations with $\mathrm{A} \beta_{40}(r=0.04 ; p=0.758)$, PAI-1 ( $\left.r=0.19 ; p=0.176\right)$, or hsCRP $(r=0.21 ; p=0.147)$ (Fig. 2$)$.

Finally, a backwards linear regression analysis was performed to estimate the independent effect of different variables on the decrease in cIMT. The following variables were introduced into the model: the absolute changes in BMI, HOMA-IR, hsCRP, PAI-1, A $\beta_{40}$, and STWEAK, as well as age. Of these variables, only the changes in BMI $(\beta=0.389 ; p=0.005)$ and $\operatorname{sTWEAK}(\beta=-0.358$; $p=0.009)$ were associated with the decrease in cIMT $\left(R^{2}=0.313 ; F=9.348 ; p<0.001\right)$.

\section{Discussion}

Our study shows that in obese women, both RYGB and SG induce a similar increase in circulating sTWEAK concentrations that is associated with the decrease in cIMT. In contrast, no changes in $A \beta_{40}$ were observed. Although obesity surgery decreased the circulating 


\section{Obesity Facts}
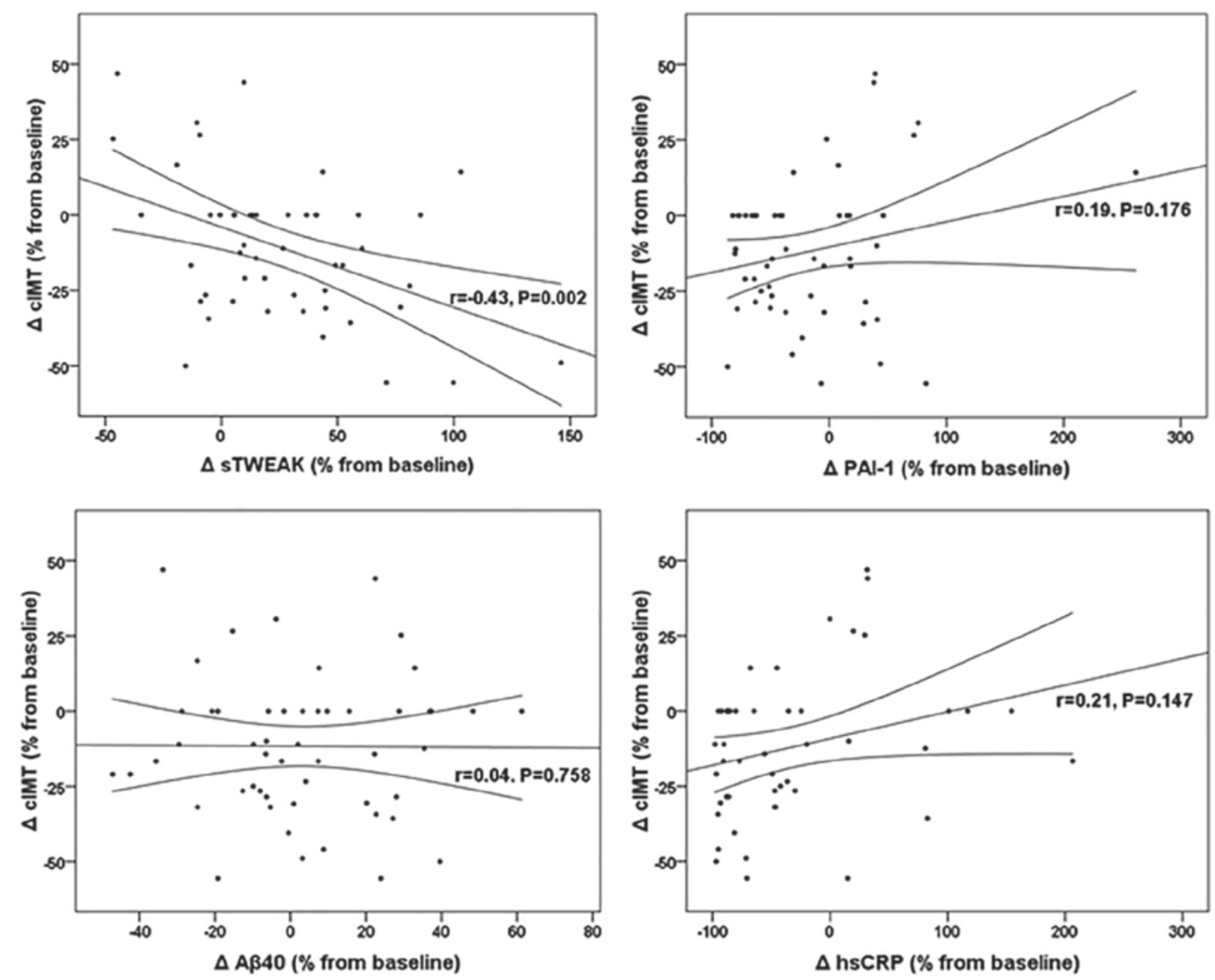

Fig. 2. Correlations of changes in cIMT with those of sTWEAK (soluble tumor necrosis factor-like weak inducer of apoptosis), amyloid- $\beta(1-40)\left(A \beta_{40}\right)$, plasminogen-activator inhibitor type 1 (PAI- 1 ), and high-sensitivity C-reactive protein (hSCRP). $\Delta$ represents the change in the variable at the end of follow-up expressed as $\%$ from baseline. cIMT, carotid intima-media thickness.

concentrations of hsCRP and PAI-1, these two factors did not correlate with the changes observed in cIMT.

There are many known pathways by which sTWEAK interacts with the endothelium and the atherosclerotic process. TWEAK upregulates the cell surface expression of adhesion molecules such as ICAM-1 and E-selectin in endothelial cells [29], as well as chemokine expression and secretion by both vascular smooth muscle cells and macrophages [30]. In vivo experiments developed in ApoE knockout mice demonstrated that administration of recombinant TWEAK increased atherosclerotic plaque size, and this effect was prevented by administration of a TWEAK-blocking antibody [30]. Furthermore, both genetic deletion of TWEAK and TWEAK-blocking antibody administration in these mice reduced plaque size and inflammatory response, thus enhancing plaque stability [31]. TWEAK inhibition also ameliorated diabetes-driven atherosclerosis through the attenuation of STAT1 activation, a critical inflam- 
matory pathway by which hyperglycemia contributes to the pathogenesis of diabetic complications [32].

The circulating concentrations of sTWEAK are a useful marker of endothelial integrity and the atherosclerotic process, as arterial pathological conditions upregulate $\mathrm{Fn} 14$ expression [17] - the TWEAK receptor - and consequently decrease its circulating levels [18]. sTWEAK concentrations are independently and negatively associated with cIMT in asymptomatic subjects, gradually decreasing as the number of atherosclerotic plaques increases [33] and with the presence of coronary artery disease [20,34]. Severely obese subjects - who have a high cardiovascular disease risk - show reduced sTWEAK levels, which increase after obesity surgery in the majority of patients [35]. Accordingly, we found an increase in sTWEAK in obese women after obesity surgery, and as a novel finding, such an increase correlated with the improvement in cIMT.

$\mathrm{A} \beta_{40}$ has been associated with an increased risk for cardiovascular mortality in patients with coronary artery disease [27], and cohort-based studies revealed that $A \beta_{40}$ was significantly and independently associated with arterial stiffness progression, incident subclinical atherosclerosis, and incident coronary heart disease [26]. However, we did not find any changes in this marker after bariatric surgery and no association with the decrease in cIMT. It is possible that $A \beta_{40}$ is not sensitive enough in the setting of severely obese patients, or that our sample size was too small to detect differences in this marker.

Hypofibrinolysis is considered a precipitating factor in the occurrence of clinical cardiovascular events through thrombus formation on atherosclerotic plaques, and it is also involved in the development of atherosclerotic plaque [15]. However, a recent study has shown controversial data regarding PAI-1 after obesity surgery, with different results in the short and medium term after surgery and also between patients with and those without diabetes [36]. Although circulating PAI-1 concentrations positively correlated with cIMT both in young and in adult obese subjects $[37,38]$, the addition of PAI- 1 to other classic cardiovascular risk factors did not improve prediction of high cIMT in a large cohort of healthy young adults [39]. Accordingly, our data show that PAI-1 concentrations, albeit being reduced after both RYGB and SG, were not associated with the decrease in cIMT observed after bariatric surgery.

A similar pattern, consisting of a decrease after both surgical techniques but no correlation with the overall change in CIMT, was found for hsCRP, a well-characterized inflammation marker [40]. Although hsCRP has been shown to decrease after obesity surgery [41], it is possible that it may not be as sensitive as sTWEAK. In fact, the latter better predicted the presence of carotid atherosclerotic plaques in patients with chronic kidney disease [42] and in asymptomatic subjects [33].

The major limitation of this study is the lack of randomization in the allocation of the patients to the groups submitted to bariatric surgery. The strict application of our hospital protocol biased BMI, insulin resistance, and inflammatory markers towards higher values in the subgroup of patients submitted to RYGB. This bias might also explain the lower baseline STWEAK concentrations found in these women. In addition, because men were excluded in order to limit the variability in basal cIMT, thereby facilitating the finding of differences between groups, our results are only applicable to women.

In conclusion, RYGB and SG in obese women induce a similar increase in circulating STWEAK 1 year after surgery, which parallels the decrease in cIMT.

\section{Acknowledgments}

We thank the nurse staff of the Department of Endocrinology and Nutrition for their help with anthropometric assessment and blood sampling of the patients.

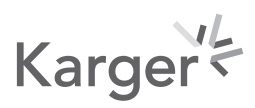




\section{Statement of Ethics}

Written informed consent was obtained from every participant, and the study was approved by the institutional ethics review board of our institution. All procedures performed involving human participants were in accordance with the ethical standards of the institutional and/or national research committee and with the 1964 Helsinki Declaration and its later amendments or comparable ethical standards.

\section{Disclosure Statement}

The authors declare no conflict of interest.

\section{Funding Sources}

Supported by Grant AES/ISCIII - PI18/00132 and FIBioHRyC EM03/2010. CIBERDEM and CIBEROBN are also initiatives of Instituto de Salud Carlos III, co-financed by the European Development Regional Fund "A Way to Achieve Europe" (ERDF).

\section{Author Contributions}

The authors have made substantial contributions to the conception and design of the study (J.A.B. and J.I.B.-C.), acquisition of data (J.M.G.-M., E.A., M.I., G.d.I.P., and J.G.) or analysis and interpretation of data (M.A.L., H.F.E.-M., J.A.B., and J.I.B.-C.), or drafting the article or revising it critically for important intellectual content (J.M.G.-M., J.G., M.A.L., H.F.E.-M., J.A.B., and J.I.B.-C.). All authors approved the final submitted manuscript.

\section{References}

1 Basterra-Gortari FJ, Bes-Rastrollo M, Ruiz-Canela M, Gea A, Sayón-Orea C, Martínez-González MA. Trends of obesity prevalence among Spanish adults with diabetes, 1987-2012. Med Clin (Barc). 2019 Mar;152(5):181-4.

2 Finucane MM, Stevens GA, Cowan MJ, Danaei G, Lin JK, Paciorek CJ, et al.; Global Burden of Metabolic Risk Factors of Chronic Diseases Collaborating Group (Body Mass Index). National, regional, and global trends in body-mass index since 1980: systematic analysis of health examination surveys and epidemiological studies with 960 country-years and 9.1 million participants. Lancet. 2011 Feb;377(9765):557-67.

3 Whitlock G, Lewington S, Sherliker P, Clarke R, Emberson J, Halsey J, et al.; Prospective Studies Collaboration. Body-mass index and cause-specific mortality in 900,000 adults: collaborative analyses of 57 prospective studies. Lancet. 2009 Mar;373(9669):1083-96.

4 Berrington de Gonzalez A, Hartge P, Cerhan JR, Flint AJ, Hannan L, MacInnis RJ, et al. Body-mass index and mortality among 1.46 million white adults. N Engl J Med. 2010 Dec;363(23):2211-9.

5 Sjöström L. Bariatric surgery and reduction in morbidity and mortality: experiences from the SOS study. Int ] Obes. 2008 Dec;32(S7 Suppl 7):S93-7.

6 Sjöström L, Gummesson A, Sjöström CD, Narbro K, Peltonen M, Wedel H, et al.; Swedish Obese Subjects Study. Effects of bariatric surgery on cancer incidence in obese patients in Sweden (Swedish Obese Subjects Study): a prospective, controlled intervention trial. Lancet Oncol. 2009 Jul;10(7):653-62.

7 Sjöström L, Narbro K, Sjöström CD, Karason K, Larsson B, Wedel H, et al.; Swedish Obese Subjects Study. Effects of bariatric surgery on mortality in Swedish obese subjects. N Engl J Med. 2007 Aug;357(8):741-52.

8 Lupoli R, Di Minno MN, Guidone C, Cefalo C, Capaldo B, Riccardi G, et al. Effects of bariatric surgery on markers of subclinical atherosclerosis and endothelial function: a meta-analysis of literature studies. Int J Obes. 2016 Mar; 40(3):395-402.

9 Vlachopoulos C, Xaplanteris P, Aboyans V, Brodmann M, Cífková R, Cosentino F, et al. The role of vascular biomarkers for primary and secondary prevention. A position paper from the European Society of Cardiology Working Group on peripheral circulation: Endorsed by the Association for Research into Arterial Structure and Physiology (ARTERY) Society. Atherosclerosis. 2015 Aug;241(2):507-32. 


\begin{tabular}{|c|c|}
\hline \multicolumn{2}{|l|}{ Obes Facts $2020 ; 13: 321-330$} \\
\hline DOI: 10.1159/000507087 & $\begin{array}{l}\text { (c) } 2020 \text { The Author(s). Published by S. Karger AG, Base } \\
\text { www.karger.com/ofa }\end{array}$ \\
\hline
\end{tabular}

10 Gómez-Martin JM, Aracil E, Galindo J, Escobar-Morreale HF, Balsa JA, Botella-Carretero JI. Improvement in cardiovascular risk in women after bariatric surgery as measured by carotid intima-media thickness: comparison of sleeve gastrectomy versus gastric bypass. Surg Obes Relat Dis. 2017 May;13(5):848-54.

11 Gómez-Martin JM, Balsa JA, Aracil E, Insenser M, Priego P, Escobar-Morreale HF, et al. Circulating adiponectin increases in obese women after sleeve gastrectomy or gastric bypass driving beneficial metabolic changes but with no relationship with carotid intima-media thickness. Clin Nutr. 2018 Dec;37(6 Pt A):2102-6.

12 Gómez-Martin JM, Balsa JA, Aracil E, Cuadrado-Ayuso M, Rosillo M, De la Peña G, et al. Beneficial changes on plasma apolipoproteins A and B, high density lipoproteins and oxidized low density lipoproteins in obese women after bariatric surgery: comparison between gastric bypass and sleeve gastrectomy. Lipids Health Dis. 2018 Jun;17(1):145.

13 Van Gaal LF, Mertens IL, De Block CE. Mechanisms linking obesity with cardiovascular disease. Nature. 2006 Dec;444(7121):875-80.

14 Hansson GK. Inflammation, atherosclerosis, and coronary artery disease. N Engl J Med. 2005 Apr;352(16): 1685-95.

15 Kohler HP, Grant PJ. Plasminogen-activator inhibitor type 1 and coronary artery disease. N Engl J Med. 2000 Jun;342(24):1792-801.

16 Winkles JA. The TWEAK-Fn14 cytokine-receptor axis: discovery, biology and therapeutic targeting. Nat Rev Drug Discov. 2008 May; (5):411-25.

17 Muñoz-García B, Martín-Ventura JL, Martínez E, Sánchez S, Hernández G, Ortega L, et al. Fn14 is upregulated in cytokine-stimulated vascular smooth muscle cells and is expressed in human carotid atherosclerotic plaques: modulation by atorvastatin. Stroke. 2006 Aug;37(8):2044-53.

18 Blanco-Colio LM. TWEAK/Fn14 Axis: A Promising Target for the Treatment of Cardiovascular Diseases. Front Immunol. 2014 Jan; 5:3.

19 Chicheportiche Y, Bourdon PR, Xu H, Hsu YM, Scott H, Hession C, et al. TWEAK, a new secreted ligand in the tumor necrosis factor family that weakly induces apoptosis. J Biol Chem. 1997 Dec;272(51):32401-10.

20 Jelić-Ivanović Z, Bujisić N, Spasić S, Bogavac-Stanojević N, Spasojević-Kalimanovska V, Kotur-Stevuljević J. Circulating sTWEAK improves the prediction of coronary artery disease. Clin Biochem. 2009 Sep;42(13-14): 1381-6.

21 Moreno JA, Dejouvencel T, Labreuche J, Smadja DM, Dussiot M, Martin-Ventura JL, et al. Peripheral artery disease is associated with a high CD163/TWEAK plasma ratio. Arterioscler Thromb Vasc Biol. 2010 Jun;30(6): 1253-62.

22 De Meyer GR, De Cleen DM, Cooper S, Knaapen MW, Jans DM, Martinet W, et al. Platelet phagocytosis and processing of beta-amyloid precursor protein as a mechanism of macrophage activation in atherosclerosis. Circ Res. 2002 Jun;90(11):1197-204.

23 Van De Parre TJ, Guns PJ, Fransen P, Martinet W, Bult H, Herman AG, et al. Attenuated atherogenesis in apolipoprotein E-deficient mice lacking amyloid precursor protein. Atherosclerosis. 2011 May;216(1):54-8.

24 Chisari M, Merlo S, Sortino MA, Salomone S. Long-term incubation with beta-amyloid peptides impairs endothelium-dependent vasodilatation in isolated rat basilar artery. Pharmacol Res. 2010 Feb;61(2):157-61.

25 Sonkar VK, Kulkarni PP, Dash D. Amyloid $\beta$ peptide stimulates platelet activation through RhoA-dependent modulation of actomyosin organization. FASEB J. 2014 Apr;28(4):1819-29.

26 Stamatelopoulos K, Sibbing D, Rallidis LS, Georgiopoulos G, Stakos D, Braun S, et al. Amyloid-beta (1-40) and the risk of death from cardiovascular causes in patients with coronary heart disease. J Am Coll Cardiol. 2015 Mar;65(9):904-16.

27 Stamatelopoulos K, Mueller-Hennessen M, Georgiopoulos G, Sachse M, Boeddinghaus J, Sopova K, et al. Amyloid- $\beta$ (1-40) and Mortality in Patients with Non-ST-Segment Elevation Acute Coronary Syndrome: A Cohort Study. Ann Intern Med. 2018 Jun;168(12):855-65.

28 Alberti KG, Eckel RH, Grundy SM, Zimmet PZ, Cleeman JI, Donato KA, et al.; International Diabetes Federation Task Force on Epidemiology and Prevention; Hational Heart, Lung, and Blood Institute; American Heart Association; World Heart Federation; International Atherosclerosis Society; International Association for the Study of Obesity. Harmonizing the metabolic syndrome: a joint interim statement of the International Diabetes Federation Task Force on Epidemiology and Prevention; National Heart, Lung, and Blood Institute; American Heart Association; World Heart Federation; International Atherosclerosis Society; and International Association for the Study of Obesity. Circulation. 2009 Oct;120(16):1640-5.

29 Harada N, Nakayama M, Nakano H, Fukuchi Y, Yagita H, Okumura K. Pro-inflammatory effect of TWEAK/Fn14 interaction on human umbilical vein endothelial cells. Biochem Biophys Res Commun. 2002 Dec;299(3):48893.

30 Muñoz-García B, Moreno JA, López-Franco O, Sanz AB, Martín-Ventura JL, Blanco J, et al. Tumor necrosis factor-like weak inducer of apoptosis (TWEAK) enhances vascular and renal damage induced by hyperlipidemic diet in ApoE-knockout mice. Arterioscler Thromb Vasc Biol. 2009 Dec;29(12):2061-8.

31 Sastre C, Fernández-Laso V, Madrigal-Matute J, Muñoz-García B, Moreno JA, Pastor-Vargas C, et al. Genetic deletion or TWEAK blocking antibody administration reduce atherosclerosis and enhance plaque stability in mice. J Cell Mol Med. 2014 Apr;18(4):721-34.

32 Fernández-Laso V, Sastre C, Méndez-Barbero N, Egido J, Martín-Ventura JL, Gómez-Guerrero C, et al. TWEAK blockade decreases atherosclerotic lesion size and progression through suppression of STAT1 signaling in diabetic mice. Sci Rep. 2017 Apr;7(1):46679. 
33 Fernández-Laso V, Sastre C, Valdivielso JM, Fernández E, Martín-Ventura JL, Egido J, et al. Soluble TWEAK levels predict the presence of carotid atherosclerotic plaques in subjects free from clinical cardiovascular diseases. Atherosclerosis. 2015 Apr;239(2):358-63.

34 Chorianopoulos E, Jarr K, Steen H, Giannitsis E, Frey N, Katus HA. Soluble TWEAK is markedly upregulated in patients with ST-elevation myocardial infarction and related to an adverse short-term outcome. Atherosclerosis. 2010 Jul;211(1):322-6.

35 Maymó-Masip E, Fernández-Veledo S, Garcia España A, Vázquez-Carballo A, Tinahones FJ, García-Fuentes E, et al. The rise of soluble TWEAK levels in severely obese subjects after bariatric surgery may affect adipocytecytokine production induced by TNF $\alpha$. J Clin Endocrinol Metab. 2013 Aug;98(8):E1323-33.

36 Mossberg KE, Pournaras DJ, Welbourn R, le Roux CW, Brogren H. Differential response of plasma plasminogen activator inhibitor 1 after weight loss surgery in patients with or without type 2 diabetes. Surg Obes Relat Dis. 2017 Jan;13(1):53-7.

37 Gilardini L, Pasqualinotto L, Di Matteo S, Caffetto K, Croci M, Girola A, et al. Factors associated with early atherosclerosis and arterial calcifications in young subjects with a benign phenotype of obesity. Obesity (Silver Spring). 2011 Aug;19(8):1684-9.

38 Khalil A, Huffman MD, Prabhakaran D, Osmond C, Fall CH, Tandon N, et al.; New Delhi Birth Cohort. Predictors of carotid intima-media thickness and carotid plaque in young Indian adults: the New Delhi birth cohort. Int J Cardiol. 2013 Aug;167(4):1322-8.

39 Raiko JR, Oikonen M, Wendelin-Saarenhovi M, Siitonen N, Kähönen M, Lehtimäki T, et al. Plasminogen activator inhitor-1 associates with cardiovascular risk factors in healthy young adults in the Cardiovascular Risk in Young Finns Study. Atherosclerosis. 2012 Sep;224(1):208-12.

40 Ridker PM, Hennekens CH, Buring JE, Rifai N. C-reactive protein and other markers of inflammation in the prediction of cardiovascular disease in women. N Engl J Med. 2000 Mar;342(12):836-43.

41 Askarpour M, Khani D, Sheikhi A, Ghaedi E, Alizadeh S. Effect of Bariatric Surgery on Serum Inflammatory Factors of Obese Patients: A Systematic Review and Meta-Analysis. Obes Surg. 2019 Aug;29(8):2631-47.

42 Fernández-Laso V, Méndez-Barbero N, Valdivielso JM, Betriu A, Fernández E, Egido J, et al. Soluble TWEAK and atheromatosis progression in patients with chronic kidney disease. Atherosclerosis. 2017 May;260:130-7. 